Western University

Scholarship@Western

Electrical and Computer Engineering

Electrical and Computer Engineering

Publications

Department

Fall 9-24-2017

Development and Grasp Analysis of a Sensorized Underactuated

Finger

Mahyar Abdeetedal

Amazon

Mehrdad Kermani Ph.D., P.Eng.

Western University, mkermani@eng.uwo.ca

Follow this and additional works at: https://ir.lib.uwo.ca/electricalpub

Part of the Computer Engineering Commons, and the Electrical and Computer Engineering Commons

Citation of this paper:

Abdeetedal, Mahyar and Kermani, Mehrdad Ph.D., P.Eng., "Development and Grasp Analysis of a Sensorized Underactuated Finger" (2017). Electrical and Computer Engineering Publications. 548.

https://ir.lib.uwo.ca/electricalpub/548 


\title{
Development and Grasp Analysis of a Sensorized Underactuated Finger
}

\author{
Mahyar Abdeetedal and Mehrdad R. Kermani ${ }^{1}$
}

\begin{abstract}
This paper presents the design and evaluation of a new sensorized underactuated self-adaptive finger. Our design incorporates a two degrees-of-freedom (DOF) parallel based underactuated mechanism with an embedded load cell for contact force measurement and a trimmer potentiometer to acquire the joint variables. Integration of the sensors leads to tactile feedback fidelity without compromising the finger size and complexity which results in efficient and robust functionality. The particular rounded shape of the distal phalanx and high equilibrium position enable the finger to deliver both precision and power grasps. The effectiveness of our design is verified theoretically and through experimental results demonstrating its shape adaptability, and tactile capability.
\end{abstract}

\section{INTRODUCTION}

An industrial gripper is mostly used to manipulate only one or a few objects of similar shape. Small changes in the object shape or weight require the gripper to be modified [1]. There are industrial applications, such as agricultural harvesting in which target crops in a field are not necessarily the same. In the design of robotic hands, task adaptation capability usually correlates with complex kinematic structures with a high number of degrees of freedom, which may increase the size, control complexity and weight of the device. In addition, practical cases in which the operation varies from one object to the other, grasp configuration is different for each grasp scenario. Planning new grasp configurations requires contact force and location to fulfill the task objectives toward accurate object placement and damage avoidance. Addressing all mentioned challenges usually adds up to the gripper size and complexity. Tight conditions on space requirements, on the other hand, demand for a compact gripper design. In this paper, we propose an underactuated finger design which provides tactile feedback fidelity without compromising its size and complexity.

We need a mechanism which can passively adapt to different objects shape, without requiring adding more actuators and/or sophisticated control strategies. When a robotic mechanism has fewer actuators than the degrees of freedom, it is known as an underactuated mechanism. An underactuated robotic hand provides passive motions demanded by the object geometry. In an underactuated power grasp, the robotic hand wraps around the object and provides a robust grasp. The underactuated hand can be designed in a way to be capable of applying precision grasp, in which finger tip is in contact with the object. Underactuated fingers can be based on tendon-actuated mechanism or linkages. Most of tendon-actuated mechanisms are limited to small grasping

\footnotetext{
${ }^{1}$ Mahyar Abdeetedal and Mehrdad R. Kermani are with Faculty of Electrical and Computer Engineering, Western University, London, Ontario, Canada mabdeete@uwo.ca, mkerman2@uwo.ca
}

forces that lead to friction and elasticity [2]. There are a number of important underactuation approaches for robotic hands, e.g., eigen-grasps [3], parallel structure based [2] and adaptive synergies [4], [5]. In this paper, the development and evaluation of a sensorized link-driven finger are considered due to its robust structure.

The form adaptability of link-driven underactuated fingers comes at the price of closing motion dictated by the shape of the object. Therefore, without additional sensors contact positions are unknown. Tactile sensors can be used to acquire contact forces and positions [2], [6]. Typically, tactile sensors, also known as robot skins consists of an array of sensors covering areas of a robot to provide contact positions and forces [7]. Robot skins are usually sophisticated and prohibitively costly [8]. Another approach is adopted in [9] which takes advantage of negative torque compensation at the inter phalanx joints of the finger to roughly estimate the contact position. In this paper, by embedding the load cell and trimmer potentiometer in the finger structure the contact forces and positions are obtained. Our design allows for a low cost, yet a reasonably efficient alternative to tactile sensors. The main contributions of this paper are as follows

- A novel approach is introduced for obtaining tactile information. Our approach is based on the integration of potentiometer and load cell data. Both experimentally and theoretically, it is shown that the suggested approach is capable of contact position estimation.

- The Jacobian matrix, containing transmission matrix and contact model is derived. The new formulation for transmission matrix considers both prismatic and revolute actuation of an underactuated mechanism.

- A new sensorized underactuated finger is designed and 3D printed. The embedded load cell facilitates the grasp of fragile objects such as egg. Furthermore, unknown object centroid approximation is implemented via contact estimation, joint variable measurement, and selfadaptation of the finger.

The structure of this paper is as follows: Section II presents the proposed contact estimation method. Section III provides the underactuated finger design. Section IV studies the validity of the presented approach via designing and building an underactuated finger.

\section{Contact Point Estimation}

To define the relevant velocity kinematics and force transmission properties of a robotic hand, the fingers Jacobian $J$ is needed. The Jacobian matrix is defined as a mapping from the transmitted contact forces and moments to the load of the joints. Kinematically, the Jacobian matrix maps the joint 


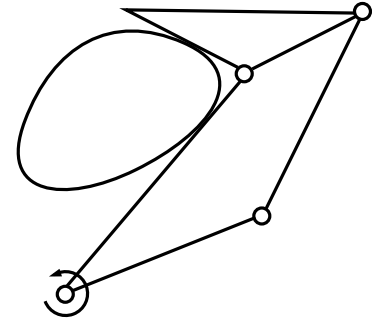

(a)

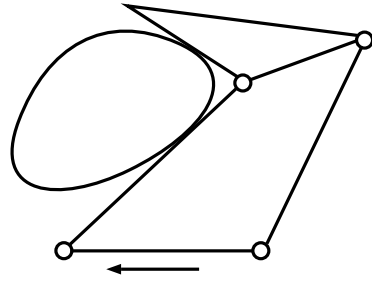

(b)
Fig. 1. Two different ways of actuating the 2-DOF underactuated finger. (a) actuation using a revolute joint, and (b) actuation using prismatic joint.

velocities to the twists of the hand at contact frames. Usually, in the underactuation literature, finger characterization is done independently from the object being grasped [2]. In this section, we integrate two important concepts into the Jacobian matrix definition. First, both cases of revolute and prismatic joint for actuating the underactuation is formulated. Figure 1 shows the two different ways of actuating a twoDOF underactuated finger by a revolute joint or a prismatic joint. In both cases of actuation, the geometry of the object causes the closure of the finger. In a link-driven mechanism, each phalanx activates its next phalanx until full finger closure around the object. Second, contact model is considered which is important for obtaining grasp capabilities. Three common models for contact are considered here.

Matrix $\tilde{J}$ relates various velocity quantities of all contact points as, $v_{c, \text { fin }}=\tilde{J} \dot{q}$ where $v_{c, \text { fin }}$ is the contact twist on the finger, $q$ is the phalanx joint coordinates. Let $\theta_{i}$ be the $i^{\text {th }}$ joint angle, $l_{i}$ be the $i^{\text {th }}$ link length, and $c_{i}$ be the position of the contact point $i$. By developing Plücker coordinates of the axes of the joints [10] for linkage based underactuated manipulator shown in Fig. 2 matrix $\tilde{J}$ can be obtained as follow,

$$
\tilde{J}=\left[\begin{array}{cccc}
c_{1} & 0 & \ldots & 0 \\
0 & 0 & \ldots & 0 \\
1 & 0 & \ldots & 0 \\
\hline n_{21} & c_{2} & \ldots & 0 \\
t_{21} & 0 & \ldots & 0 \\
1 & 1 & \ldots & 0 \\
\hline \ldots & \ldots & \ldots & \ldots \\
\ldots & \ldots & \ldots & \ldots \\
\ldots & \ldots & \ldots & \ldots \\
\hline n_{n 1} & n_{n 2} & \ldots & c_{n} \\
t_{n 1} & t_{n 2} & \ldots & 0 \\
1 & 1 & \ldots & 1
\end{array}\right]
$$

where $n_{i j}=c_{i}+\sum_{k=j}^{i-1} l_{k} \cos \left(\sum_{m=k+1}^{i} \theta_{m}\right), j<i$ and $t_{i j}=$ $\sum_{k=j}^{i-1} l_{k} \sin \left(\sum_{m=k+1}^{i} \theta_{m}\right), j<i$. Note that a rotation about the first axis $\left(q_{1}\right)$ does not change the kinematic configuration in a linkage based underactuated system.

Another important matrix for underactuated grasp characterization is transmission matrix, $T$. Matrix $T$ relates the input velocity vector, $\dot{\theta}$, to the derivatives of the phalanx

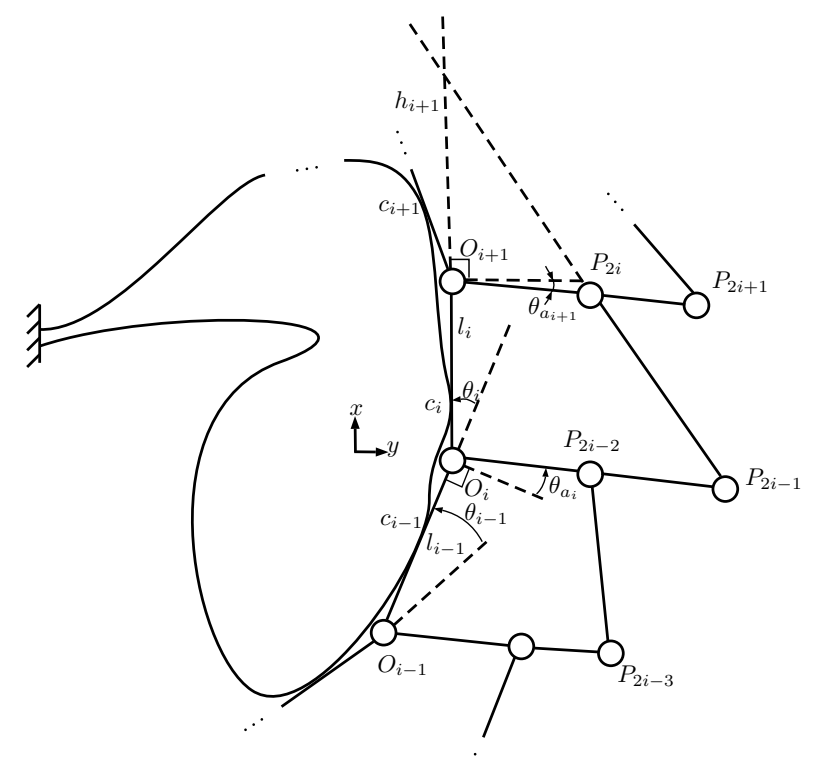

Fig. 2. Detailed modeling of a linkage-driven finger in contact with a general object with unknown geometry.

joint coordinates, $\dot{q}$.

$$
\dot{q}=T \dot{\theta}
$$

The development procedure of matrix $T$ is discussed in literature [2]. We modified matrix $T$ to be as follows,

$$
T^{T}=\left[\begin{array}{cc}
\rho & 0_{n-1}^{T} \\
-\frac{h_{2}}{h_{2}+l_{1}} & \\
\cdots & I_{n-1} \\
-\prod_{i=2}^{n} \frac{h_{i}}{h_{i}+l_{i-1}} &
\end{array}\right]
$$

where $\rho \dot{\theta}_{1}$ is the arc that first joint variable makes, and $h_{i}$ is the signed distance between point $O_{i}$ and the intersection of $\left(\mathrm{O}_{i-1} \mathrm{O}_{i}\right)$ and $\left(\mathrm{P}_{2 i-2} \mathrm{P}_{2 i-3}\right)$ (see Fig. 2). In this matrix, we included the actuation type of the underactuation mechanism by the first component, $\rho$. For the case of actuating the underactuated finger by a revolute joint $\rho=1$, and for prismatic actuation $\rho \simeq c_{1}$. Note that a curve in the plane can be approximated by connecting a finite number of points on the curve using line segments to create a polygonal path. The length of each linear segment can be calculated using the Pythagorean theorem in Euclidean space. Here, the arc that is shaped by each phalanx rotation was approximated with a line equivalent to the prismatic joint variable change.

There are three different contact models that are commonly used in grasp modeling: point contact without friction, hard finger, and soft finger. To obtain the complete Jacobian matrix, we have to include each particular contact model to select suitable components of the contact twists which are transmitted between the finger $\left(v_{i, f i n}\right)$ and the object $\left(v_{i, o b j}\right)$. The contacts model can be expressed as, $H\left(v_{c, f i n}-v_{c, o b j}\right)=$ 0 where $H=\operatorname{Blockdiag}\left(H_{1}, \ldots, H_{n_{c}}\right), H_{i}$ can be chosen from Table $\mathrm{I}$ according to the $i^{\text {th }}$ contact model. The contact model matrix $H$ selects suitable components of the contact twist and 
TABLE I

SELECTION MATRIX FOR PLANAR CONTACT $i$.

\begin{tabular}{ll}
\hline \hline Model & $H_{i}$ \\
\hline \hline Point contact without friction & {$\left[\begin{array}{lll}1 & 0 & 0\end{array}\right]$} \\
Hard and soft finger & {$\left[\begin{array}{lll}I_{2 \times 2} & 0\end{array}\right]$} \\
\hline \hline
\end{tabular}

sets them to zero. Now we can obtain the complete Jacobian matrix as,

$$
J=H \tilde{J} T
$$

The goal here is to estimate the location of the contact points using the provided definition for the Jacobian matrix. Using a dual view of the Jacobian matrix definition, $\tau=J^{T} F$ where $\tau$ is the actuation vector and $F$ is the contact forces vector, the equation for the first generalized joint torque of the finger is derived as follows,

$$
\tau_{a}=\rho c_{1} f_{c_{1}}
$$

where $\tau_{a}$ is the generalized actuation torque of the first (actuated) joint, and $f_{c_{1}}$ is the first contact force. For other joints,

$$
\tau_{k}=\left(-\prod_{i=2}^{k} \frac{h_{i}}{h_{i}+l_{i-1}} n_{k 1}+c_{k}\right) f_{c_{k}}
$$

where $\tau_{k}$ is the actuation torque of the $k^{\text {th }}$ joint, and $f_{c_{k}}$ is the normal contact force on the $k^{\text {th }}$ phalanx. In quasistatic assumption, the acceleration of mechanism is not considered. Therefore, at each state of grasping from the moment of first phalanx contact to the complete closure of the finger by the distal phalanx contact, all forces and torques are in balance. In a link-driven underactuated robot in which low stiffness springs are used to hold the structure, the input torque vector exerted by the actuator and springs is obtained as,

$$
\tau=\left[\begin{array}{c}
\tau_{a} \\
\tau_{2}=-K_{2} \Delta \theta_{2} \\
\cdots \\
\tau_{n}=-K_{n} \Delta \theta_{n}
\end{array}\right]
$$

where $K_{i}$ is the $i^{\text {th }}$ spring constant, $\tau_{a}$ is the generalized actuation torque input, and $\tau_{i}$ is the $i^{\text {th }}$ joint torque. It is assumed that finger makes contacts with the object by every phalanxes which is the case for most power grasps. It is also assumed that first phalanx was subjected to the first contact at the moment of gripper closure. This assumption is valid for the link-driven underactuated finger, since the next phalanx is activated when the distal phalanx is in contact. Using [5], (6), and (7), we can obtain contact position estimation. In our proposed design, trimmer potentiometer provides $\Delta \theta_{i}$ for the $i^{\text {th }}$ joint and the $i^{\text {th }}$ embedded load cell provides the contact force on the $i^{\text {th }}$ phalanx. By equating (5) and (7) at the moment of first contact and repeating the procedure for other phalanxes using (6), all contact locations become available.

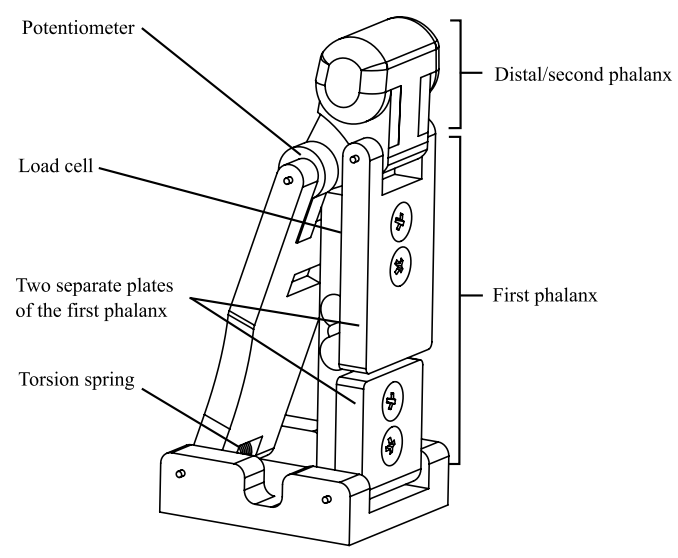

Fig. 3. Underactuated finger packed with a load cell and a potentiometer. Two separate plates attached to the load cell form the first phalanx. The rounded fingertip allows finger to be bent in precision grasping.

\section{Underactuated FInger DESIGN}

A parallel underactuated finger is designed and built as a test-bed to validate the method for contact location estimation. The design is kept small and straightforward regarding manufacturing. It is 3D printable, and it has few parts to assemble. We aim for packing the finger with position and force sensors while keeping the design compact. The 3D design model of the finger is shown in Fig. (3). The total length of the finger is $8 \mathrm{~cm}$, its largest width is $3 \mathrm{~cm}$, the width at its tip is $2 \mathrm{~cm}$. For this design, $l_{1}=5.5 \mathrm{~cm}, h_{1} \simeq$ $3.4 \mathrm{~cm}$ (at $\theta_{3}=0$ ). The positive distance $h$ at rest position is considered to be equal to the length of the distal phalanx. Hence, any contact point on the distal phalanx falls below the equilibrium point which results in finger closure. The first joint can rotate $60^{\circ}$, and the second joint can rotate $80^{\circ}$.

Mechanical limits are considered to allow a preloading of the spring to prevent any undesirable motion of the distal phalanx due to its weight and/or inertial effects, and also to avoid hyperflexion of the finger. Springs are meant for keeping the finger from incoherent motion until the grasp sequence is completed. Since springs oppose the actuator force, we have considered springs with the smallest stiffness possible, however, sufficient to keep the finger from collapsing. The tip of the finger is designed to be rounded which can roll on the object. Therefore, bending of the finger happens in precision grasping as well as power grasping.

In a linkage underactuated finger, one joint variable can provide the values of other joint variables since they are kinematically dependent. For measuring a joint variable, we placed a trimmer potentiometer which is fixed from one side to the first phalanx, and the other end attached to the distal phalanx. The joint rotation causes the potentiometer to rotate and read different resistance. Reading the second joint variable is enough for obtaining the first joint variable.

Placing a load cell in the finger design is a challenging task. First, we want to consume minimal space for the sensor in the finger. Second, load cell has to be placed in a position 


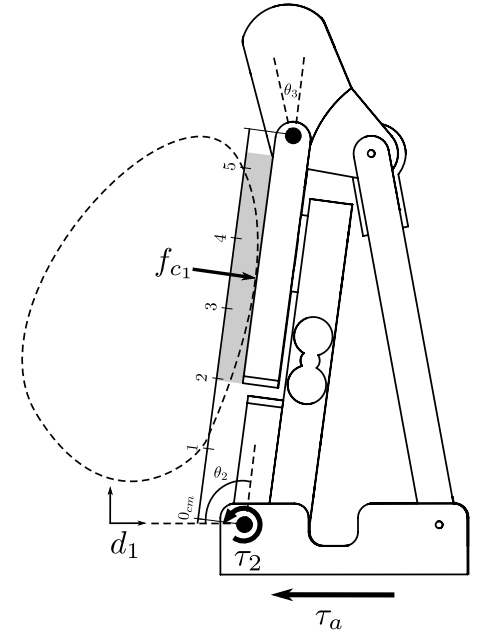

Fig. 4. Generalized actuation, $\tau_{a}$, moves the finger toward the object. Contact force $f_{c_{1}}$ causes finger closure which is reacted by spring and results torque $\tau_{2} . d_{1}$ is the first joint variable, $\theta_{2}$ is the first joint variable, and $\theta_{3}$ is the second joint variable. The gray area (on the second plate) is the length that contact force is measurable (60\% of the first phalanx length).

to be able to read meaningful force data. We address these challenges by considering the load cell as a part of the first phalanx (see Fig. 4). Two separate plates of the first phalanx are attached to the load cell to provide room for the strain of the load cell. The shown gray area is the length that contact force is measurable (60\% of the first phalanx length). The lower plate is considered to have the smallest possible size. During a grasping, the load cell starts reading contact force while the finger is bending. The acquired contact force is directly related to the stiffness of the spring and its strain. When the finger is fully bent, the load cell is fixed at its bottom and continues reading contact force.

\section{Results}

The objective of the experiments was to prove the capability of the gripper in conducting power grasping as well as precision grasping. Moreover, contact force regulation was done to demonstrate the ability of the finger in dealing with fragile objects. Additionally, it was shown that the designed finger was able to estimate the contact location. We stepped further ahead and accomplished the object centroid and shape approximation.

\section{A. Experimental Setup}

Kuka Light-Weight Robot (LWR) IV and CRS Robotics underactuated gripper were used for evaluating the performance of the designed underactuated fingers. The load cells in the fingers and the trimmer potentiometers at their joints were used for acquiring data (see Fig. 57. To exploit the control capabilities of Kuka LWR in parallel use with peripheral tools and sensors, we used the open-source KUKA UI (https://github.com/mahyaret/KUKA-UI).

Load cells were calibrated for measuring the contact points using a ATI 6-axis force/torque sensor. The calibration was done for different contact points including precision and power grasping. The data from potentiometer was acquired

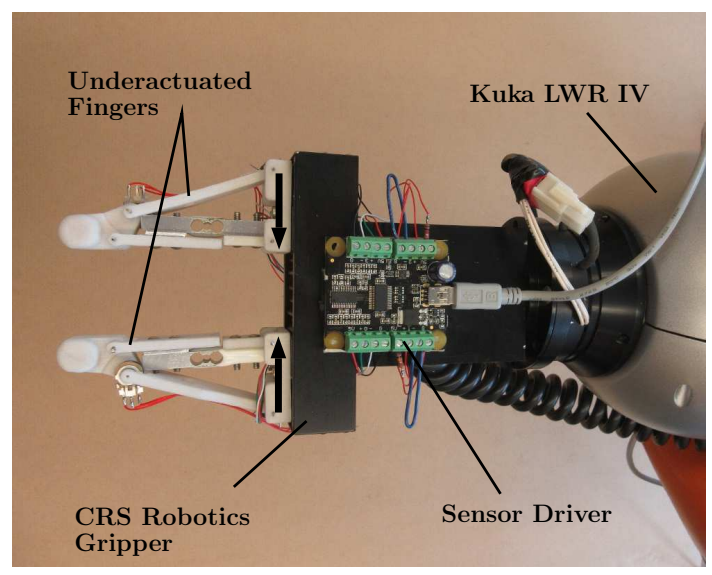

Fig. 5. Experimental hardware setup. A single driver is used for collecting data from two load cells and two potentiometers.

using the same driver for load cells which was interfaced to the developed software.

The matrix $H$, and $\tilde{J}$ for the two fingers actuated by CRS Robotics gripper were developed using Table II (1), respectively as follow,
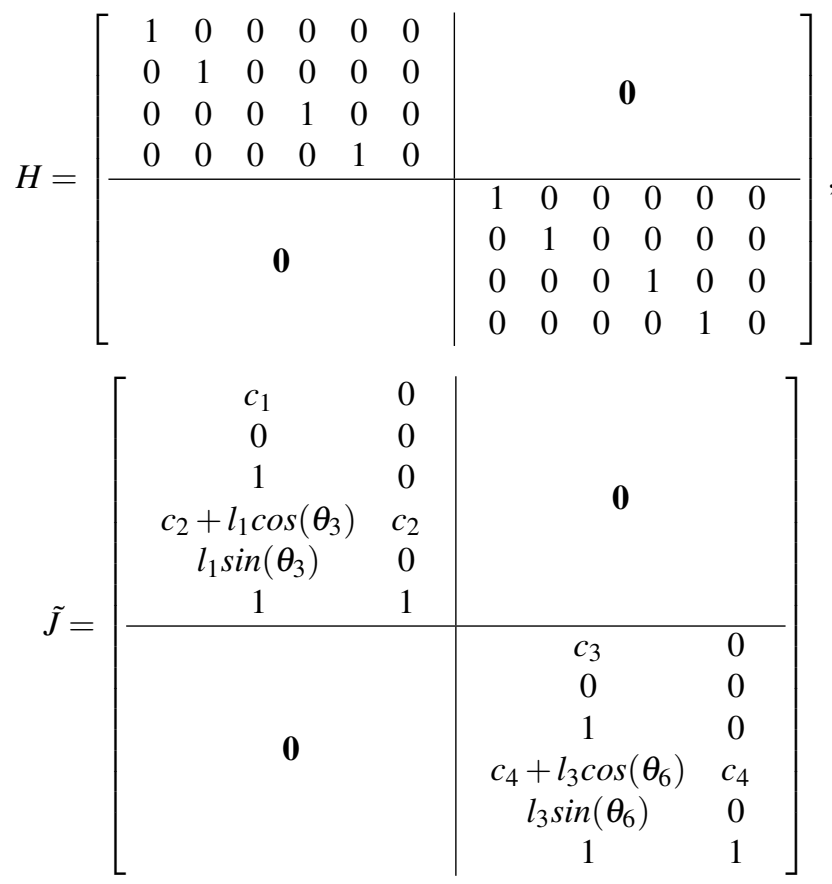

where $c_{1}$ and $c_{2}$ are the first and second contact points on one finger, $c_{3}$ and $c_{4}$ are third and forth contact points on the other finger, $\theta_{3}$ is the second joint variable on one finger, $\theta_{6}$ is the second joint variable on the other finger, and $l_{1}=l_{3}=$ $5.5 \mathrm{~cm}$ for the fingers. The relation between the input velocity vector to the derivatives of the joint variables, transmission matrix in (3), is as follows,

$$
\left[\begin{array}{c}
\dot{\theta}_{2} \\
\dot{\theta}_{3} \\
\dot{\theta}_{5} \\
\dot{\theta}_{6}
\end{array}\right]=\left[\begin{array}{cc|cc}
c_{1} & -\frac{h_{1}}{h_{1}+l_{1}} & & \mathbf{0} \\
0 & 1 & & \\
\hline & \mathbf{0} & c_{3} & -\frac{h_{2}}{h_{2}+l_{3}} \\
& & 0 & 1
\end{array}\right]\left[\begin{array}{c}
\dot{d}_{1} \\
\dot{\theta}_{3} \\
\dot{d}_{4} \\
\dot{\theta}_{6}
\end{array}\right]
$$




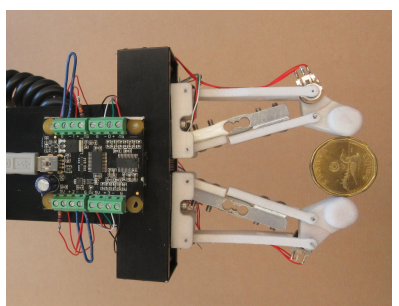

(a)

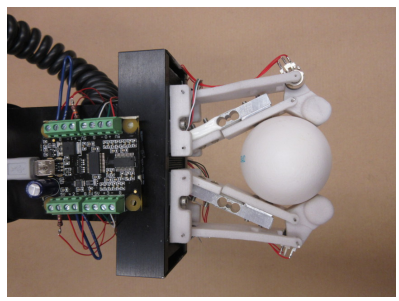

(c)

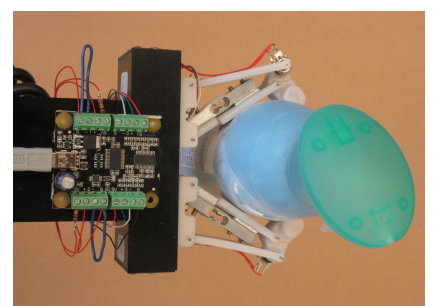

(b)

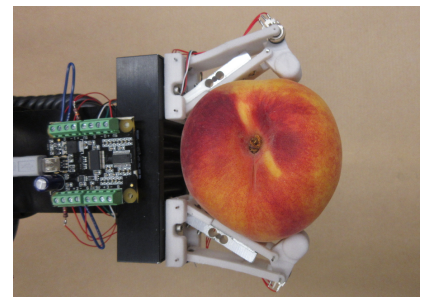

(d)
Fig. 6. Adaptability of the designed finger. (a) Precision grasp of a coin. (b) Power grasp of a spray bottle. (c) Power grasp of an egg. (d) Power grasp of a peach.

where $h_{1}=h_{2} \simeq 3.4 \mathrm{~cm}$ for first and second fingers at rest position $\left(\theta_{3}=\theta_{6}=0\right), d_{1}$ and $d_{4}$ are prismatic joint variables of the fingers.

\section{B. Shape Adaptability}

To test the adaptability of the gripper, we grasped a broad range of objects. In general, the underactuated fingers performed well. Examples of grasps are shown in Fig. 6. As mentioned before, the rounded design of the distal phalanx causes bending of the finger even in precision grasping. Figure 6a shows a precision grasp, in which the distal phalanx was bent. The experiments carried out with the use of load cell sensors show that these sensors were required in many fragile objects grasping tasks. The hand was able to grasp very fragile objects, such as an egg (see Fig. 6c). The small width of the fingers and the size of the hand facilitated the manipulation in constrained situations such as harvesting fruit and vegetable.

\section{Force Control}

Since CRS Robotics gripper was used in our experiments, fingers were actuated using the prismatic joints. Force control was obtained based on the force feedback provided by the load cell. Since the strain gauge based force sensor noise is unavoidable, a PID controller enhanced with Kalman filter was used to regulate the contact forces. The force regulations for $f_{c_{1}}$ shown in Fig. 4 at $3.5 N, 5.5 N$, and $7 N$, as well as the respective joint variables, are illustrated in Fig. 7. The velocity at impact was transferred into a high force overshoot. The overshoot in the force control was also due to static friction of the joints and the stress of the torsion spring. We believe that the fusion of the position data and force readings can better deal with impact force and contact detection. This conjecture however, requires further investigation.

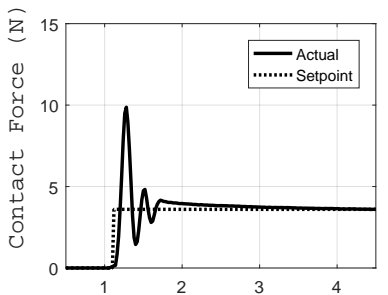

(a)

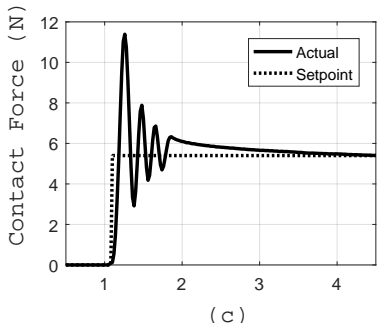

(c)

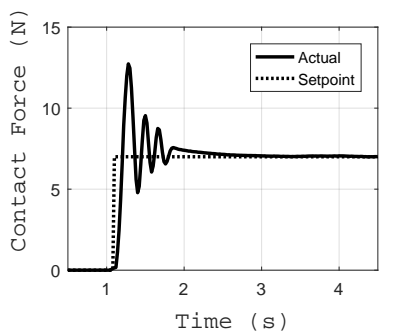

(e)

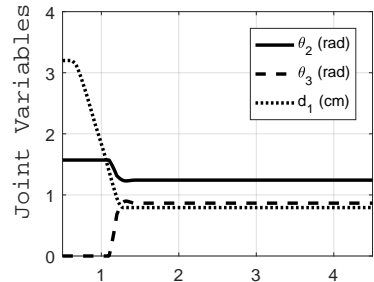

(b)

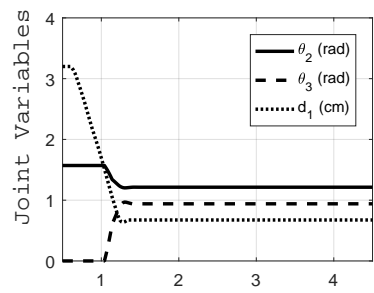

(d)

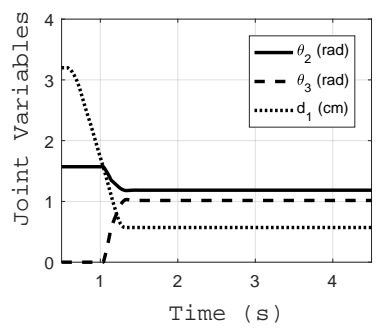

(f)
Fig. 7. Force control and joint variable. $d_{1}$ is the first joint variable, $\theta_{1}$ is the first unactuated joint variable, and $\theta_{2}$ is the second joint variable. (a), and (b) Force regulation for $3.5 \mathrm{~N}$ and the second joint angle. (c), and (d) Force regulation for $5.5 \mathrm{~N}$ and the second joint angle. (e), and (f) Force regulation for $7 N$ and the second joint angle.

\section{Contact Point Estimation}

Using (5) and (7), the equation for joints torque vector of the finger was derived as follows,

$$
K_{2} \Delta \theta_{2}=c_{1}^{2} f_{c_{1}}, K_{5} \Delta \theta_{5}=c_{3}^{2} f_{c_{3}}
$$

where $K_{2} \Delta \theta_{2}$ and $K_{5} \Delta \theta_{5}$ are equal to the actuation torque of the two finger, $f_{c_{1}}$ and $f_{c_{3}}$ are the first contact forces on the fingers. By regulating the second joint variables $\left(\theta_{2}\right.$ and $\left.\theta_{5}\right)$ and measuring the forces $\left(f_{c_{1}}\right.$ and $\left.f_{c_{3}}\right)$ using load cells, (8) can be verified. The scaled first phalanx is shown in Fig. 4. The second joint variable was regulated at $1.2 \mathrm{rad}$ while first phalanx was in contact with the object at different contact points. The respective contact force for each contact point was measured. The result is illustrated in Fig. 8 which shows the validity of the contact estimation in (8).

In our design, we did not used a load cell in the distal phalanx to keep overall design shorter. Since the distal phalanx was $64 \%$ shorter than the first phalanx, the contact point on the distal phalanx had a small torsional effect. This small leverage of the second contact force enabled us to assume its position to be on the distal phalanx edge without compromising much accuracy. Further experiments using different general convex and concave shapes validated this simplification.

To validate the simplification for the distal phalanx contact 


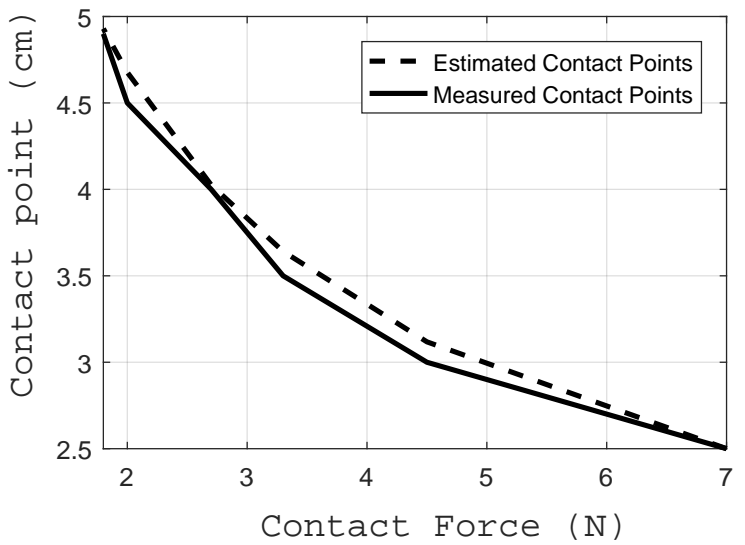

Fig. 8. Contact points estimation using the measured force on the first phalanx of the finger.

point estimation, we needed to quantify the amount of uncertainty it may cause in object position estimation and grasp analysis. The object frame is usually fixed to the centroid of the object to develop Grasp matrix or to be used for object placement. We designed an experiment in which finding the centroid of the object was desired. Different objects with general shapes were 3D printed and grasped. The contact forces on the first phalanx of each finger estimated using (8), and the distal phalanx contact points are considered to be on the edge of the phalanx.

Kinematic dependency in all joints of the underactuated finger, as well as contact points knowledge, were used to approximate the grasped object with a polygon/polyhedron. Let us assume that $\left\{c_{i}=\left(x_{i}, y_{i}\right)\right\}_{i=0}^{n_{c}} \subset R^{2}$ is a closed approximation polygon in the plane, the vertices are ordered counter clockwise. The centroid is given by,

$$
p=\frac{1}{6 A}\left[\begin{array}{c}
\sum_{i=0}^{N-1}\left(x_{i}+x_{i+1}\right)\left(x_{i} y_{i+1}-x_{i+1} y_{i}\right) \\
\sum_{i=0}^{N-1}\left(y_{i}+y_{i+1}\right)\left(x_{i} y_{i+1}-x_{i+1} y_{i}\right)
\end{array}\right]
$$

where $A=\frac{1}{2} \sum_{i=0}^{N-1}\left(x_{i} y_{i+1}-x_{i+1} y_{i}\right)$, is the area that is enclosed by the polygon. Having all contact points on both fingers, we approximated the object shape by a 4-gon, and its centroid was obtained using (9). Figure 9 shows the validation of contact points and object center estimation for various convex and concave objects. As it can be seen in this figure, the simplification caused small uncertainty (about a maximum of $8 \%$ ) in centroid estimation.

\section{COnClusion And Future Work}

In this paper, Jacobian matrix, containing contact model and transmission matrix for the underactuated system were obtained. The centroid of the object was obtained by kinematically adapting the underactuated finger and sensors data. An underactuated finger packed with force and position sensors was designed and manufactured to validate the introduced theory. Such compact design with tactile feedback proved to be practical in a wide range of manipulation tasks. Contact force controllability widened the range of possible

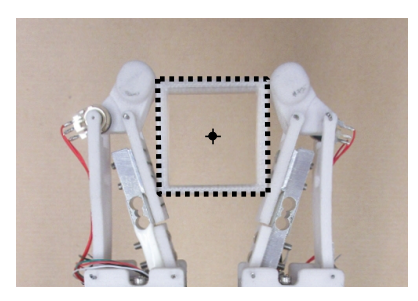

(a)

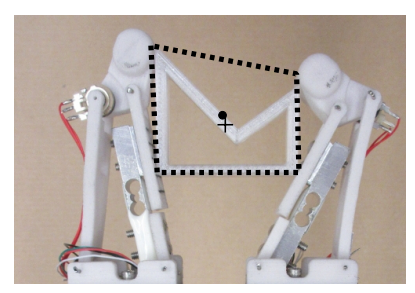

(c)

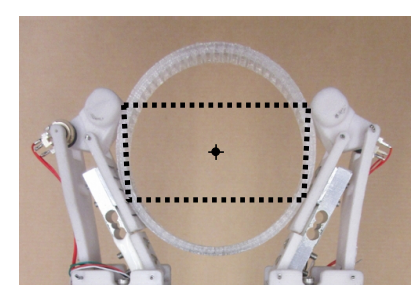

(b)

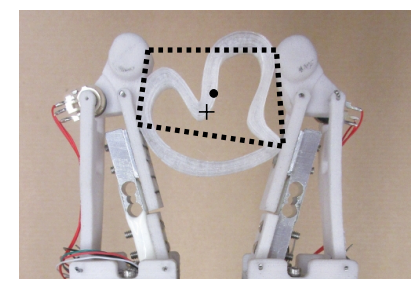

(d)
Fig. 9. Object centroid estimation. - is the estimated centroid, and + is the actual mass center. (a) Estimated square centroid and its mass center coinciding . (b) Estimated circle centroid and its mass center coinciding . (c) Estimated concave polygon centroid and its mass center. (d) Estimated concave object centroid and its mass center.

target object from fragile to tough. The future work will be using the position sensor and fusion of its data with force data to have a better force regulation in the presence of nonlinear/anisotropic joint friction, and noise which is an unavoidable case for all force sensors. A video is attached to this submission of which a high resolution version can be found at: https: / / youtu.be/yruMRA9iLS8

\section{REFERENCES}

[1] P. O. Hugo, "Industrial grippers: State-of-the-art and main design characteristics," in Grasping in Robotics. Springer, 2013, pp. 107131.

[2] L. Birglen, T. Laliberté, and C. M. Gosselin, Underactuated robotic hands. Springer, 2007, vol. 40.

[3] M. Ciocarlie, C. Goldfeder, and P. Allen, "Dexterous grasping via eigengrasps: A low-dimensional approach to a high-complexity problem," in Robotics: Science and Systems Manipulation WorkshopSensing and Adapting to the Real World. Citeseer, 2007.

[4] M. G. Catalano, G. Grioli, A. Serio, E. Farnioli, C. Piazza, and A. Bicchi, "Adaptive synergies for a humanoid robot hand," in 2012 12th IEEE-RAS International Conference on Humanoid Robots (Humanoids 2012). IEEE, 2012, pp. 7-14.

[5] M. Gabiccini, E. Farnioli, and A. Bicchi, "Grasp analysis tools for synergistic underactuated robotic hands," The International Journal of Robotics Research, p. 0278364913504473, 2013.

[6] A. M. Dollar, L. P. Jentoft, J. H. Gao, and R. D. Howe, "Contact sensing and grasping performance of compliant hands," Autonomous Robots, vol. 28, no. 1, pp. 65-75, 2010.

[7] F. L. Hammond, R. K. Kramer, Q. Wan, R. D. Howe, and R. J. Wood, "Soft tactile sensor arrays for micromanipulation," in 2012 IEEE/RSJ International Conference on Intelligent Robots and Systems. IEEE, 2012, pp. 25-32.

[8] Z. Kappassov, J.-A. Corrales, and V. Perdereau, "Tactile sensing in dexterous robot handsreview," Robotics and Autonomous Systems, vol. 74, pp. 195-220, 2015.

[9] B. Belzile and L. Birglen, "Stiffness analysis of underactuated fingers and its application to proprioceptive tactile sensing," IEEE/ASME Transactions on Mechatronics, vol. 21, no. 6, pp. 2672-2681, 2016.

[10] J. K. Davidson and K. H. Hunt, Robots and screw theory: applications of kinematics and statics to robotics. Oxford University Press on Demand, 2004. 